Rapid Reviews COVID-19

\title{
Review 1: "Repurposed antiviral drugs for COVID- 19; interim WHO SOLIDARITY trial results"
}

Alberto Borobia ${ }^{1}$, Antonio J Carcas ${ }^{2}$

${ }^{1}$ La Paz University Hospital, Clinical Pharmacology, Spain,

${ }^{2}$ Clinical Pharmacology Department. La Paz University Hospital. School of Medicine. Universidad Autónoma de Madrid. IdiPAZ

Published on: Nov 11, 2020

DOI: $10.1162 / 2 \mathrm{e} 3983 f 5 . b f f b 9 b b 7$

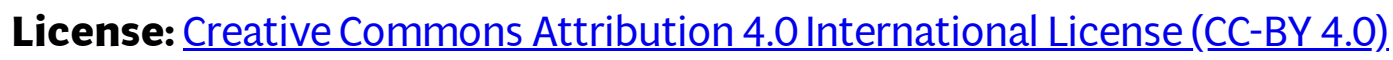




\section{$\underline{\text { RR:C19 Evidence Scale rating by reviewer: }}$}

- Reliable. The main study claims are generally justified by its methods and data. The results and conclusions are likely to be similar to the hypothetical ideal study. There are some minor caveats or limitations, but they would/do not change the major claims of the study. The study provides sufficient strength of evidence on its own that its main claims should be considered actionable, with some room for future revision.

$* * * * * * * * * * * * * * * * * * * * * * * * * * * * * * * * * * * * * * *$

\section{Reasoning for Strength of Evidence Rating:}

The main claims made by the authors are justified, mainly because the lack of evidence of effectiveness is enough to not recommend the use of any drug. In addition, results for Hydroxychloroquine, Lopinavir/Ritonavir are consistent with those of Recovery trial $(1,2)$. The data of Interferon- $\beta 1$ a do not include relevant differences in its $95 \%$ CI. However, there are some factors that can affect reliability of results and especially the size effects found. This could be relevant for Remdesivir. Decision-makers should consider results on Hydroxychloroquine, Lopinavir/Ritonavir and Interferon- $\beta 1 \mathrm{a}$ as actionable. In the case of Remdesivir, the conclusion is weaker.

\section{Review:}

We agree with the authors in many of the strengths of this study, being a very good experience in the design and development of multinational, extremely pragmatic trial in a pandemic context and to be applied to any hospital around the world. However, this study shows limitations related to the design, reporting and analysis of the results.

\section{General aspects}

- Authors should better describe the procedures for obtaining the patients' consent to enter the trial and follow-up.

- Both the manuscript and the protocol are very scarce in details about procedures for interim analysis: how and why the timing for interim analysis and release results were decided.

\section{Population and randomization}


- The age of the population included in the study is lower than the age described in cohorts from Spain $(3,4)$, Italy (5), UK (6) and US (7). This could influence the validity of the conclusions, especially in the case of Hydroxychloroquine and Interferon- $\beta 1 \mathrm{a}$, where some heterogeneity is shown in relation to age.

- Details of the randomization procedure should be more clearly described, as it is a bit confusing.

- $\quad$ One of the main problems we see is related to the pre-randomization patient selection. What it is intriguing is the different percentage of patients assigned to the different drugs in relation with the severity of the disease. Patients on oxygen at entry or already ventilated were $75.9 \%$ of those treated with Remdesivir, $63.6 \%$ of those treated with Hydroxychloroquine, $62.3 \%$ of those with Lopinavir/Ritonavir and 76.5\% of those treated with Interferon- $\beta 1$ a. Similar differences are shown in relation with lung lesions. Also, the proportion of patients using corticosteroids in each "trial" is different (table 2, Suppl): 47.8\% in "Remdesivir trial," 14.8\% in "Hydroxychloroquine trial," 22.6\% in "Lopinavir/Ritonavir trial" and 47.9\% in the "Interferon- $\beta 1$ a trial." We would expect that these percentages would be distributed similarly between drugs.

- As per Figure 1, there is a substantial number of patients with no reported or no reached last follow-up. This could bias the results, mainly those of Remdesivir and Interferon- $\beta 1 \mathrm{a}$.

- It is not clear if these results can be applied to patients in early treatment of the disease, as it is difficult to know at which stage of the disease the patients were.

\section{Treatments}

- Hydroxychloroquine dose is very high. Authors should justify this and discuss the potential influence in the results, including safety issues and/or potential adverse drug reactions related to these high doses.

- Authors should describe how oral drugs were administered in ventilated patients. It is important to account for the time from symptom initiation to start of treatment. In a clinical trial evaluating antiviral drugs we would expect that these drugs are effective only if administered in the first days after clinical onset. This would be especially relevant for Remdesivir. In the "Remdesivir trial” high risk groups have a tendency to increased mortality, although no statistical differences can be found. On the contrary, the lower risk groups could have benefit (IC99\%: 0.67-1.11), an estimation similar to that found in the MTA depicted in Figure 4 (IC99\%: 0.63-1.01). 


\section{Safety}

- The manuscript contains very poor description (if any) of adverse events. We recognize that with the exception of Remdesivir, this does not change the recommendation on the use of these drugs, but is a good practice to communicate as it is very important information in the benefit/risk assessment of any drug. In fact, it seems that the protocol only foresees the reporting of SUSARS.

\section{Other aspects}

- Authors give us the results by ventilation status. However, it could be very informative to provide the subgroup analysis as in Figure 4 (No O2, low/high flow O2, ventilation) for all the drugs.

- It would be nice if authors could provide raw data of the trial for other analysis or to be included in individual patient data meta-analysis.

Finally, this study highlights the difficulty of evaluating the repurposing of old drugs going directly from mainly theoretical arguments to highly pragmatic trials. We would need previous evidence of the activity and efficacy of these drugs to avoid dangerous short-ways in the development of new uses of old drugs.

\section{References}

1. RECOVERY Collaborative Group, et al. Effect of Hydroxychloroquine in Hospitalized Patients with Covid-19. N Engl J Med. 2020. PMID: 33031652

2. RECOVERY Collaborative Group, et al. Lopinavir-ritonavir in patients admitted to hospital with COVID-19 (RECOVERY): a randomised, controlled, open-label, platform trial Lancet. 2020 Oct 5;396(10259):1345-1352.

3. Borobia AM, Carcas AJ, Arnalich F et al. On Behalf Of The Covid Hulp Working Group. A Cohort of Patients with COVID-19 in a Major Teaching Hospital in Europe. J Clin Med. 2020 Jun 4;9(6):1733. doi: 10.3390/jcm9061733.

4. Berenguer J, Ryan P, Rodríguez-Baño J et al. On behalf Of COVID-19@Spain Study Group. Characteristics and predictors of death among 4035 consecutively hospitalized patients with COVID-19 in Spain. Clin Microbiol Infect. 2020 Aug 4:S1198743X(20)30431-6. doi: 10.1016/j.cmi.2020.07.024. 
5. Giacomelli A, Ridolfo AL, Milazzo L et al. 30-day mortality in patients hospitalized with COVID-19 during the first wave of the Italian epidemic: A prospective cohort study. Pharmacol Res. 2020 Aug;158:104931. doi: 10.1016/j.phrs.2020.104931. Epub 2020 May 22.

6. Annemarie B Docherty, Ewen M Harrison, Christopher A Green et al., ISARIC4C investigators. Features of 20133 UK patients in hospital with covid-19 using the ISARIC WHO Clinical Characterisation Protocol: prospective observational cohort study. BMJ. 2020 May 22;369:m1985. doi: 10.1136/bmj.m1985.

7. Richardson, S.; Hirsch, J.S.; Narasimhan, M.; Crawford, J.M.; McGinn, T.; Davidson, K.W.; Barnaby, D.P.; Becker, L.B.; Chelico, J.D.; Cohen, S.L.; et al. Presenting Characteristics, Comorbidities, and Outcomes Among 5700 Patients Hospitalized With COVID-19 in the New York City Area. JAMA 2020. 